Ahmed Mostafa, Mostafa Y. A Mostafa*, M. Abdel-Rahman, M. A. Abdel-Rahman, Emad A. Badawi*, and Y. C. Lin

\title{
The positron and mechanical parameters of a cold-worked aluminum alloy (3004) Using PALT, PADBT and $\mathrm{HV}^{* *}$
}

https://doi.org/10.1515/jmbm-2021-0032

Received Jul 23, 2021; accepted Jan 16, 2022

\begin{abstract}
In the present work, the influence of plastic deformation on the properties of a $3004 \mathrm{Al}$-alloy was studied with different techniques. Crystallite size, dislocation density, defect density, micro-strain, and stored dislocation energy are presented and compared for different three techniques. Methodology: Many techniques for detecting defects have been developed, such as the positron annihilation lifetime technique (PALT), the positron annihilation Doppler broadening technique (PADBT), and the Vickers hardness test (HV).

Implications: The positron mean lifetime value of a nondeformed sample is $173 \pm 4.8 \mathrm{ps}$, which increases until the thickness reduction reaches a $10 \%$ deformation then saturated at saturation trapping of the positron in defect states with a mean of $221 \pm 5 \mathrm{ps}$. At an S-parameter of $0.3709 \pm 0.0031$, a W-parameter of $0.5885 \pm 0.0057$ was obtained at zero deformation: this decreases until saturation at $10 \%$ deformation. Findings: A good correlation between the three techniques is observed for mean crystallite size. A good correlation was also noted between PALT and PADBT from 0 to $10 \%$ thickness reduction. HV has good correlations with PALT and PADBT from 0 to $6 \%$ thickness reduction: then a clear difference was found from 6 to $15 \%$ thickness reduction. Originality: such results confirm the fact that the used technique can effects on the obtained results in some limitations.
\end{abstract}

Keywords: $3004 \mathrm{Al}$-alloy, positron annihilation techniques, plastic deformation

\footnotetext{
^Corresponding Author: Mostafa Y. A Mostafa: Physics Department, Faculty of Science, Minia University, 61519, Egypt; Experimental Physics Department, Physics and Technology Institute, Ural Federal University, Ekaterinburg, 620002, Russia; Email: mostafa.youness@mu.edu.eg

Ahmed Mostafa, M. Abdel-Rahman, M. A. Abdel-Rahman, Emad A. Badawi: Physics Department, Faculty of Science, Minia University, 61519, Egypt
}

๖ Open Access. () 2021 A. Mostafa et al., published by De Gruyter. (cc) BY License

\section{Introduction}

Aluminum and its alloys are applied in various products and processes due to their distinct properties, such their low weight, good electrical and mechanical properties, etc. $[1,2]$. The aluminum-manganese series ( $3 \mathrm{xxx}$ ) is a one of wrought Al-alloy which is non-heat-treatable. It cannot be reinforced or strengthened by hardening precipitation. With a range of $1-2 \%$, manganese is the basic alloying element for this series. It gives these alloys plasticity: this provides them with good formability and a wide range of mechanical properties in different cold-worked states. Compared to $1 \mathrm{xxx}$ series alloys, $3 \mathrm{xxx}$ alloys have higher strength properties, particularly at high temperatures. These types of alloys are mostly strengthened by cold work (plastic deformation with compression) $[2,3]$.

PALT is considered one of the main tools utilized in materials science to study the properties of defects. PALT is used to determine defect density and size [4]. Its main advantages are that it is a very sensitive non-destructive nuclear technique and it is capable of differentiating between various types of defects (for example, vacancies, dislocations, grain boundaries, voids, etc.) [4-7]. PALT is also used to investigate free volume and permeation relationships for different polymers, metals, alloys, etc. PALT is applied to interrogate metals defects and pores, ceramics and polymers: the usefulness of PALT for the characterization of these materials is well recognized [5, 6, 8-10]. PALT has also been applied to self-assembled biomimetic systems to define stringent micelle concentration in aquatic micellar

Y. C. Lin: School of Mechanical and Electrical Engineering, Central South University, Changsha 410083, China; Light Alloy Research Institute, Central South University, Changsha 410083, China; State Key Laboratory of High Performance Complex Manufacturing, Changsha 410083, China

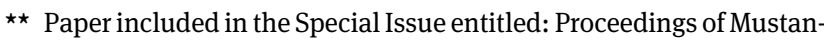
siriyah International Conference on Applied Physics - 2021 (MICAP2021) 
systems, in addition, to identify transformations in micellar geometry, aggregation numbers, and phase transitions [5].

In many new industrial applications, the radiation annihilation (electron-positron), Doppler broadening, technique (PADBT) are substantial paraphernalia in the field of materials science. Since 1970s, these techniques have been used to investigate and characterize materials point defects [11]. Recently, these techniques have been utilized in properties of intermetallic alloys, which are promising new materials for industry [11-16].

The mechanical and electrical properties of materials have significantly affected by vacancies and other lattice defects. PADBT gives the local electron momentum distribution at the annihilation site. During the process of annihilation, the momentum and energy are conserved and the two photons are mostly emitted with energies [12]. These energies are given by:

$$
E_{1,2}=m_{e}+\frac{B_{i}}{2} \pm \frac{P_{z} C}{2}
$$

where $\left(P_{z} C\right)$ is the rest mass energy of electron, $B_{i}$ is the binding energy of electron, $c$ is the light speed and $P_{z}$ is the pair momentum component (electron-positron) in the emitted gamma-rays direction. In such case, two detectors are using to observe the both annihilation photons with Doppler broadening spectroscopy [12]. Compared to the measurements taken with one detector, a sensitive improvement in the energy resolution combination is provided by the analysis of the annihilation peak, and a significant background reduction is also observed.

In order to monitor the evolution of the defects and directly observe the damage process, PADBT has been used. Upon being trapped, an annihilation of positron with an electron into double $511 \mathrm{KeV}$ photons. The detection of slight differences in the energy of the $0.511 \mathrm{MeV}$ annihilation quanta due to the electron's energy and momentum in the positron-electron annihilation is the basis of the PADBT measurements [12]. Positrons prefer to be trapped in an open volume region (e.g., vacancies, free volume, dislocations, voids, etc.), in such case, no nuclei exist $[17,18]$. In the positron-electrons annihilation process, the presented gamma rays indicate to information about the annihilation site, which makes PADBT a more sensitive probe for such investigation.

In the case of an appropriate load being applied to a substance, it causes a divergence in the material shape. This variation gives different deformations either elastic or plastic. Plastic deformation involves fracturing a restricted atomic number of bonds by dislocation movement. The mechanical properties of materials are describing and presented based on dislocations. One of the most worthy methods for the mechanical properties optimization of metals or alloys is the plastic deformation [18-21]. To achieve higher mechanical properties, plastic deformation is highly recommended for the lattice defects generation that acts as barriers to dislocation movements [21,22]. the most important fact that the elastic moduli in texture-free polycrystalline aggregates not affected by the plastic deformation.

Therefore, the metal micro-hardness is a critical parameter to define the metal strength. Hardness test presents beneficial information about the mechanical properties of material. In the present work, the Vickers hardness test is used. The Vickers hardness test is widely employed, especially for Al alloys [23-26]. Often, it is preferable to use plastic deformation than another hardness tests due to the calculations are separate and not depending on the indenter size. The Vickers hardness test can be applied for different metals and has one of the large scales amongst hardness tests. Its unit is the Vickers Pyramid Number (HV) [23-27].

In our previous work, $[18,28]$ these techniques were used separately to study the properties of different series of $\mathrm{Al}$ alloys as $6 \mathrm{xxx}$ and $5 \mathrm{xxx}$ and to distinguish between valence and core electrons of wrought alloys. In general, it was found that, as the increase of deformation, a reduction in strength and hardness is found. Secondly, the strength increases due to artificial aging with an increase in the deformation percent. Also, the defects from the alloys can be removed by the annealing process.

Therefore, the present work's aim is to use PALT (using a fast-fast coincidence) and PADBT as suitable defect characterization techniques for studying the effect of plastic deformation on the $3004 \mathrm{Al}$-alloy properties. The Vickers hardness test (HV) was used as a hardness test. The main aim of the present work is to estimate the correlation between PALT, PADBT, and Hv measurements.

\section{Materials}

A $3004 \mathrm{Al}$-alloy measuring $12 \times 12 \times 3 \mathrm{~mm}^{3}$. The $3004 \mathrm{Al}$-alloy chemical composition is presented in Table 1 . It was treated by cleaning, chemically etching, and homogenizing for six hours at $723 \mathrm{~K}$ using a furnace with non-vacuum and finally annealing process applied until room temperature. The samples after heat treatment were deformed at room temperature from 0 to $26 \%$ reduction from the original thickness utilizing a hydraulic press [29]. 
Table 1: Chemical composition of the 3004 Al-alloy

\begin{tabular}{cc}
\hline Element & $\mathrm{Wt} \%$ \\
\hline $\mathbf{M n}$ & $1.00-1.50$ \\
$\mathbf{M g}$ & $0.80-1.30$ \\
$\mathbf{F e}$ & 0.70 \\
$\mathbf{S i}$ & 0.30 \\
$\mathbf{C u}$ & 0.250 \\
$\mathbf{Z n}$ & 0.250 \\
others & 0.150 \\
$\mathbf{A l}$ & reminder \\
\hline
\end{tabular}

\section{Characterization}

PALT was performed at room temperature utilizing the system of fast-fast coincidence [30]. The time resolution of the system using a ${ }^{60} \mathrm{Co}$ source was approximately $342 \mathrm{ps}$. The positron lifetime spectrum was accumulated for 2 hours using a ${ }^{22} \mathrm{Na}$ source. About one million coincidence counts were accumulated for every spectrum. The spectrum of lifetime was analyzed as two components for lifetime using a PATFIT program.

An Ortec HPGe detector is utilized to measure the Doppler broadening line shape. It consists of a detector with an energy resolution (for ${ }^{60} \mathrm{Co}$ line $1.33 \mathrm{MeV}$ ) of 1.95 $\mathrm{keV}$, a bias supply $6595 \mathrm{kV}$, an amplifier 575 and an $8 \mathrm{k}$ MCA TRUMP. The experimental arrangement diagram setup is presented in [28]. Doppler broadening is due to the velocity distribution of the electrons that annihilating in the direction of gamma ray emission. The detector signal enters to the preamplifier input and the output from preamplifier's is fed into the amplifier. The amplifier output signal is fed into an MCA computerized.

The source positron with activity of $1 \mu \mathrm{Ci}$ of $\mathrm{NaCl}$ was vaporized from a sodium chloride hydrous solution and deposited on a fluffy foil of Kapton with a $7.5 \mu \mathrm{m}$ thickness. Radioactive substance is directly deposited on the samples; evenly, foils as separation can be applied to permit for the source to be used over and over. Also, the source may consist of one foil with radioactive substance. The source should have been thin as possible for only a small fraction of the positrons annihilate inside the source. in the present work, the deformed $3004 \mathrm{Al}$ sample with sandwich configuration is applied to guide the position. The source-sample sandwich configuration is wrapped in a thin aluminum foil. The measuring time of each sample is 1800 seconds for one spectrum.

\section{Experimental procedures}

A distribution spectrum of the number of counts versus annihilation quanta energy in a given material was collected, resulting in an almost Gaussian peak at $0.511 \mathrm{MeV}$ [28]. In the spectrum of annihilation, the shifts are related to the photo peaks broadening of $0.511 \mathrm{MeV}$, these shifts are calculated using the parameters (S- and W) [31]. The $\mathrm{S}$ - and W-parameters indicate to annihilations with the valency and the core electrons. For that, it is indispensable to observe the generation and growth of the deformationinduced defects and the chemical environment surrounding the trapping sites of the positron. The S-parameter is the area under the central part of the peak relative to the total area, while the $\mathrm{W}$-parameter is the area under the wings of the peak relative to the total area. Materials with a higher $S$ value will have more defects than materials with a lower $\mathrm{S}$ value [32].

Vickers hardness (HV) is used to determine the mechanical properties. It considers a standard method to measure the metal's hardness, particularly that with extremely hard surfaces. In such cases, the surface is affected by a gauge pressure for a criterion time length by means of a pyramid-shaped diamond. The resulting indention diagonal is measured with a microscope.

The hardness is defined as macro-, micro-, or nanoscale according to the applied forces and obtained displacements [33]. Measuring the material's macro-hardness is a rapid and straightforward procedure to obtain the bulk material mechanical data in the case of small samples. The hardness measurements were obtained with the test of Vickers hardness. Hardness is the material properties which helps material to resist plastic deformation, commonly by breakthrough. The test of Vickers hardness consists of indenting the test material with a diamond indenter, in the form of a pyramid with a quadrate base and an angle of $136^{\circ}$ between the counteractive faces. The material is exposed to a load with a range of 1 to $100 \mathrm{kgf}$. The exercised load through the Vickers hardness test was $4.9 \mathrm{~N}$. The entire load is normally applied for nearly 10 to 15 seconds. After the load removal, the two diagonals of the indentation left in the material's surface were measured with a microscope, and then the mean average calculated. The sloping surface area of the indentation was measured. The Vickers hardness is calculated by dividing the applied load by the square area of indentation [33]:

$$
H V=\frac{2 F \sin \frac{136}{2}}{d^{2}}=1.854 \frac{F}{d^{2}}
$$


where $F$ presents the load applied in kgf, d presents the average of the two diagonals in $\mathrm{mm}$ and $\mathrm{HV}$ indicates to the Vickers hardness.

\subsection{Theoretical approach to the hardness test}

Generally, through the plastic deformation, there are two dislocations types: stored statically dislocations (SSDs), that progress through uniform plastic deformation (uniform strain), and geometrically necessary dislocations (GNDs), that take place only to confirm the gradient strain (as in micro-hardness and nano-indentation tests) [34].

Plastic deformation with an effect on materials for the level of micro represents the total of reinforcement mechanisms: stress of internal friction, solid solution strengthening, strengthening of boundary grain, and strengthening dislocation [35]. With the correlation of the flow stress with hardness via the Tabor relationship, the total hardness $\left(H_{t}\right)$ is descried as the sum of the hardness of internal friction $\left(H_{i n}\right)$, hardness of solid solutions $\left(H_{s l}\right)$, hardness of dislocation density $\left(H_{d}\right)$ and hardness of grain boundary $\left(H_{g}\right)$ as follows [36, 37]:

$$
H_{t}=H_{\text {in }}+H_{s l}+H_{g}+H_{d}
$$

The effects of the grain boundary on hardness, $H_{g}$, can be negligent because the indentation happened at the grain center: the indentation size is less than the sub-grain size because no interaction between boundaries grain and dislocations is found (i.e. $H_{g}=0$ ) [36]. $H_{\text {in }}$ is independent depth because no comparing of BCC with FCC metals. Solid solutions demonstrate a more complex attitude with atoms of solute in the case of the metals non-pure via obstruction of the movement of the dislocation through plastic deformation [38]. Durst et al. when studying the solid solution effects with alloys of Ni-Fe on the hardness, they conclude two effects for the solid solution [38]. The first effect, reaction of solute atoms with dislocations (it is the effect of conventional for the solid solution) and improve the hardness with a constant amount $H_{s l}$, that related to the number of the solid atoms. The second effect, the used solid solution works as obstacles intercepting the extension of $\mathrm{GND}_{s}$, which leads to minimal storage size for dislocations; as an outcome, it impacts on $\left(H_{d}\right)$. Hence, $H_{s l}$ is a constant as $H_{\text {in }}$. Therefore, Eq. (3) can be presented for macro-hardness $\left(H_{0}\right)$ and micro-hardness $\left(H_{m i}\right)$, considering $H_{0}$ is the hardness in the $\mathrm{H}$-h curve at the plateau region and $H_{m i}$ is the hardness for the ISE zone [18]:

$$
H_{0}=H_{\text {in }}+H_{s l}+H_{S S D}
$$

$$
H_{m i}=H_{\text {in }}+H_{s l}+H_{I S E}
$$

where $H_{S S D}$ is the resistance of hardness from SSDs only and $H_{I S E}$ is the hardness resistance coming from the superposition of SSDs and GNDs [39]. Nix and Gao [40] supposed that $H_{S S D}$ and $H_{I S E}$ can be computing with the following equations:

$$
\begin{gathered}
H_{G N D}=H_{I S E}-H_{S S D} \\
H_{G N D}=H_{m i}-H_{0}
\end{gathered}
$$

where:

$$
H_{t}=H_{I S E}=H_{S S D}+H_{G N D}
$$

\section{Results and discussion}

The values of mean positron lifetime for non-deformed and deformed $3004 \mathrm{Al}$-alloys at different degrees of deformation were calculated. The mean lifetime $(\tau)$ variation with the percentage degree of deformation is given in Figure 1.

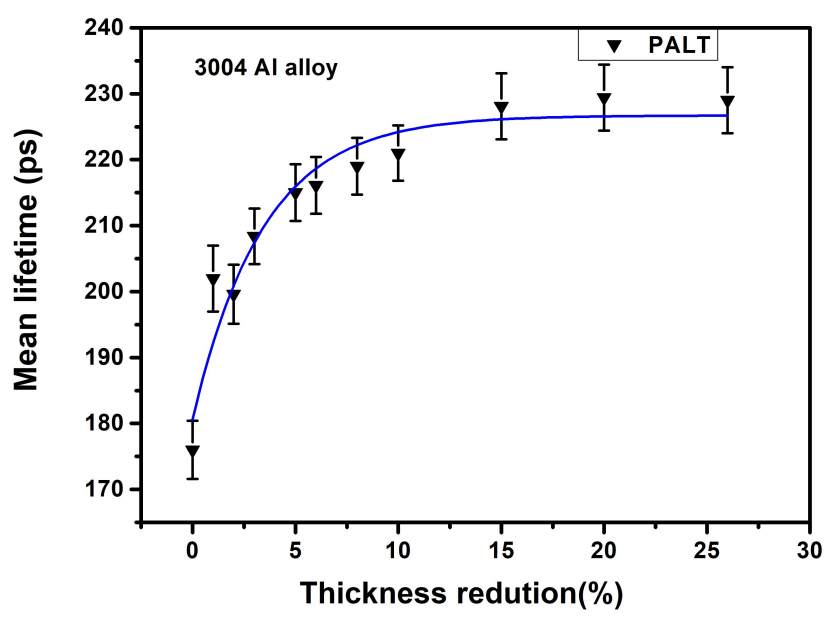

Figure 1: The positron means lifetime change with the reduction of thickness for the $3004 \mathrm{Al}$-alloy: points indicate to the experimental results and solid line gives the theoretical curve

The mean positron lifetime value, $\tau$, for the nondeformed sample is $173 \pm 4.8 \mathrm{ps}$. As the thickness reduction increased up to a $10 \%$ deformation, the values of mean lifetime increase. Above 10\% thickness reduction, the mean lifetime is approximately constant due to the saturation traps region where the positron in the defect states. For the saturated dislocation samples, the annihilation positron lifetime value of $221 \pm 5$ ps was found.

The mean crystallite size $(G)$ is presented in Table 2. By using PALT, $(G)$ and $(\tau)$ values are due to the free (bulk) 
Table 2: The mean crystallite size change with the reduction of thickness for the 3004 Al-alloy as measured by PALT

\begin{tabular}{ccc}
\hline $\begin{array}{c}\text { Deformation } \\
\text { (reduction of thickness) } \\
\%\end{array}$ & $\begin{array}{c}\text { Mean } \\
\text { crystallite } \\
\text { size }\end{array}$ & \pm \\
\hline 2 & 0.25 & 0.0125 \\
3 & 0.2 & 0.01 \\
5 & 0.17 & 0.0085 \\
6 & 0.163 & 0.00815 \\
8 & 0.153 & 0.00765 \\
10 & 0.147 & 0.00735 \\
15 & 0.13 & 0.0065 \\
20 & 0.127 & 0.00635 \\
26 & 0.129 & 0.00645 \\
\hline
\end{tabular}

lifetime $\left(\tau_{b}\right)$ and the trapped (defect) lifetime $\left(\tau_{d}\right)$ [41] as Eq:

$$
\tau=\tau_{b}+\left[\left(\tau_{d}-\tau_{b}\right) \frac{L_{+}}{G}\right]
$$

when use $S, S_{b}$ (free) and $S_{d}$ instead of $\tau, \tau_{b}$ (free) and $\tau_{d}$, respectively, one can evaluate the mean crystallite size by the values of S-parameter with PADBT [41]. Eq. (8) becomes as:

$$
S=S_{b}+\left[\left(S_{d}-S_{b}\right) \frac{L_{+}}{G}\right]
$$

where $L_{+}$, the positron diffusion length and $L_{+}=0.15 \mu \mathrm{m}$ is limited for the lifetime finite of positrons in the case of defect-free bulk $\left(\tau_{b}\right)$. This is expressed as:

$$
L_{+}=\sqrt{\tau_{b} D_{+}}
$$

where $\left(D_{+}\right)$is the diffusion coefficient of positron. The residual micro-strain $\left\langle\epsilon^{2}\right\rangle^{1 / 2}$ is given as follows [42-44]:

$$
<\epsilon^{2}>^{1 / 2}=\frac{\rho G b}{3 \sqrt{2 \pi}}
$$

where $\rho$ is the density of dislocation, $G$ is the crystallite size mean and $b$ is the Burgers vector of the Al-alloy 3004.

The parameters of Doppler broadening line shape were estimated for the non-deformed and deformed aluminum samples. The relation between the measured S- and Wparameters as points with the degree of deformation is presented in Figure 2. The S-parameter of the undeformed (annealed) sample $\left(S_{f}\right)$ is $0.3709 \pm 0.0031$, after which a rapid increase in the S-parameter from zero $\%$ to $3 \%$ deformation was followed by a slow increase from $3 \%$ to $10 \%$ deformation. Above $10 \%$ thickness reduction, the S-parameter was approximately constant in this region where saturation traps the positron in the defect states $\left(S_{t}\right.$ of $\left.0.6 \pm 0.0057\right)$. On the other hand, an increase in the S-parameter was accompanied by an exponential decrease in the $\mathrm{W}$-parameter as

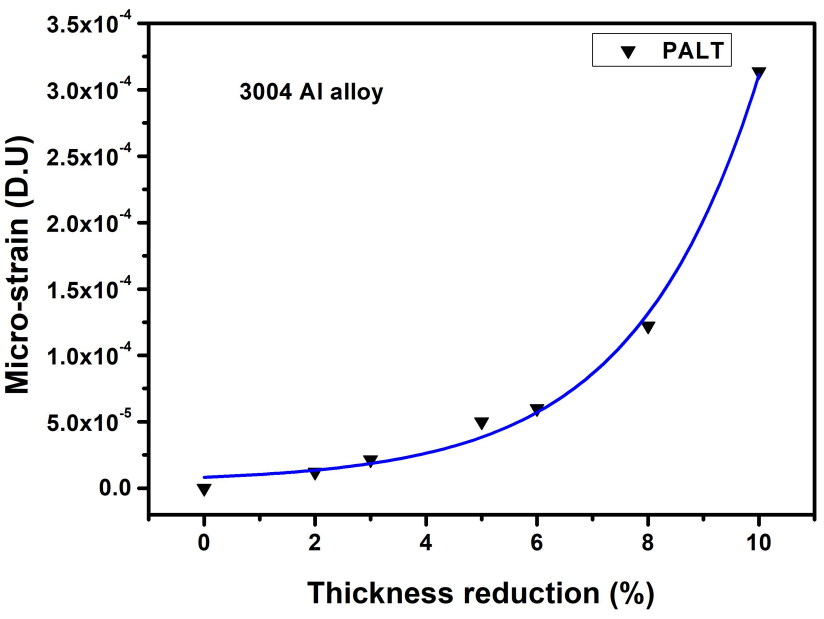

Figure 2: The micro-strain as a function of the reduction of thickness for the $3004 \mathrm{Al}$ - alloy by PALT

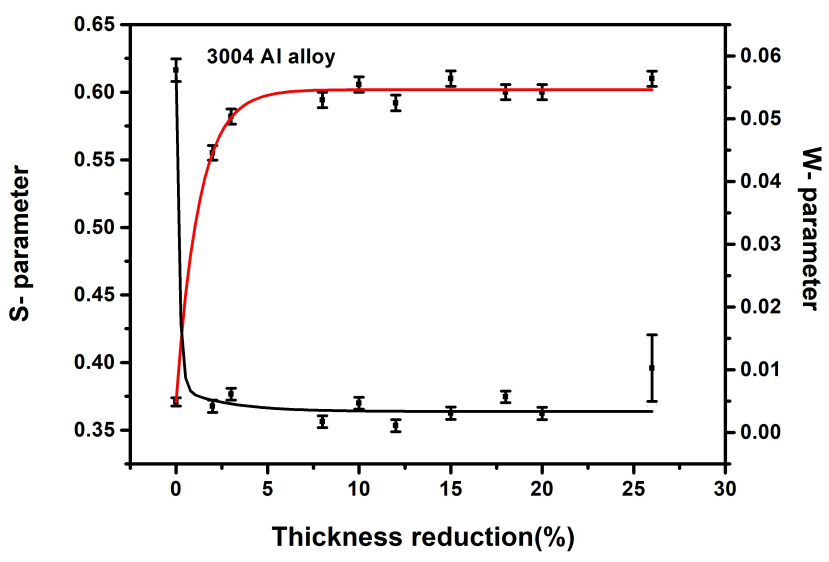

Figure 3: S- and W- parameter variations versus thickness reduction

the degree of deformation increased. At an S-parameter of $0.3709 \pm 0.0031$, a W-parameter of $0.5885 \pm 0.0057$ was obtained at zero deformation: this decreases until saturation reaches 10\% deformation, as shown in Figure 3.

Vickers hardness measurements at room temperature for the samples that plastically deformed are given in Figure 4. As shown, the total hardness, $H_{t}$, and the hardness resulting from GNDs, $H_{G N D}$, increases exponentially but slowly as the thickness reduction change from $0.0 \%$ to $6 \%$, it corresponds to $(13.50-16.40 \mathrm{HV})$ and $(0.0-3.0 \mathrm{HV})$, respectively. This result increases with the range from $6 \%$ to $15 \%$ related to (16.40-23.44 HV) and (3-10 HV), respectively. At the end, above $15 \%$ thickness reduction, it becomes constant. The mean crystallite size is determined by the following relation [41]:

$$
l=\frac{\left(H_{t}-H_{f}\right)}{\left(H-H_{f}\right)} L_{+}
$$



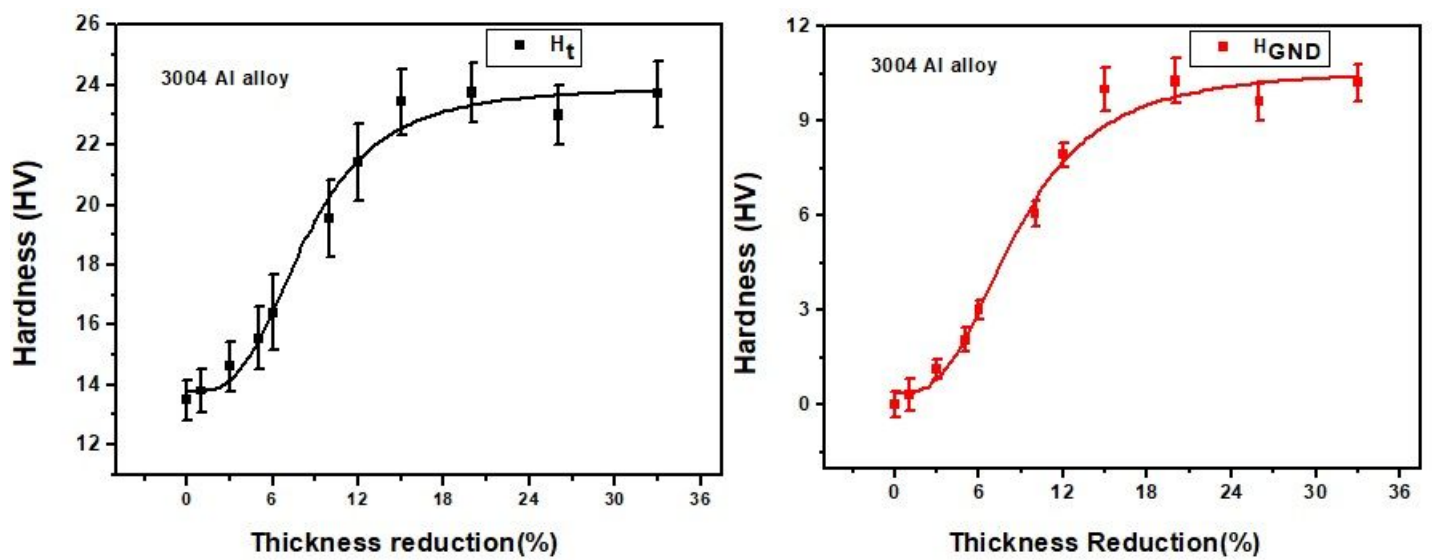

Figure 4: The total variation of hardness, $H_{t}$, and the hardness resulting from GNDs, $H_{G N D}$, in the Al-alloy 3004 with the thickness reduction

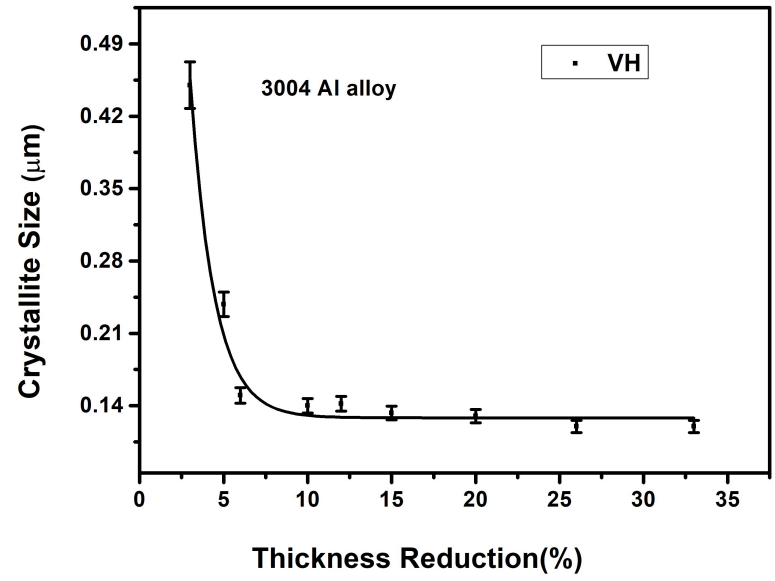

Figure 5: The mean crystallite size variation with the reduction of thickness for the 3004 Al-alloy by a Vickers hardness test

where $l$ is the mean crystallite size, $H_{f}$, Vickers hardness for the annealed sample, $H_{t}$, Vickers hardness for the saturated dislocation sample, $H$, Vickers hardness at any thickness reduction and $L_{+}$, particle diffusion length inside the alloy and equal $0.15 \mu \mathrm{m}$. In Figure 5, the mean crystallite size decreases exponentially as a function of deformation. The mean crystallite size decreases exponentially from $0.45 \mu \mathrm{m}$ to $0.14 \mu \mathrm{m}$ at thickness reduction increase from $3 \%$ to $10 \%$ respectively. Above this value of thickness reduction, the mean crystallite size becomes approximately constant.

Over the last years, many studies have shown that during micro-hardness and nano-indentation tests, hardness increases considerably as the depth of indentation (h) decreases. This is due to the GNDs effect [38, 39]. Figure 6 gives the indentation size effect (ISE). The dislocation density is given as follows [34]:

$$
H_{0}=3 \sqrt{3} \alpha G b \sqrt{\rho_{s s D_{s}}}
$$

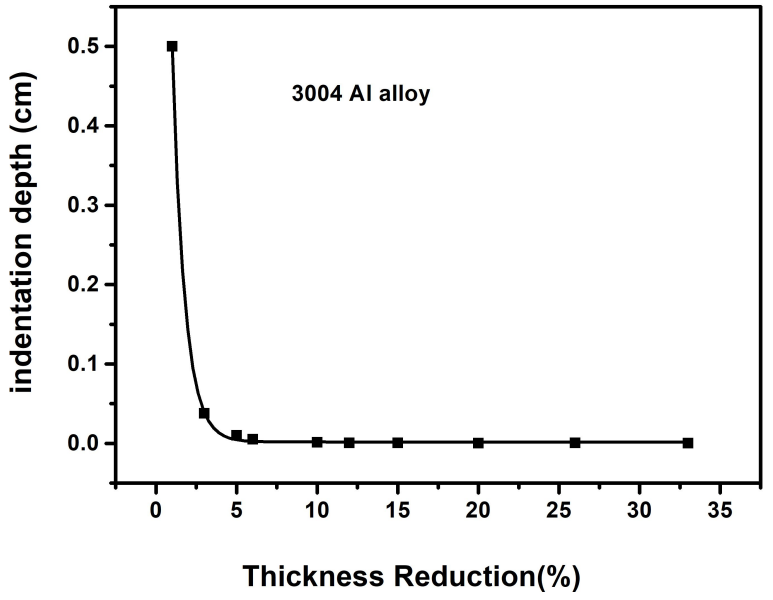

Figure 6: The indentation depth with the reduction of thickness for the 3004 Al-alloy by the Vickers hardness test results

$$
\begin{gathered}
H_{t}=3 \sqrt{3} \alpha G b \sqrt{\rho_{s s D_{s}}+\rho_{G N D}} \\
\rho_{t}=\sqrt{\rho_{s s D_{s}}+\rho_{G N D}}
\end{gathered}
$$

where $(\alpha=0.5)$ presents the parameter of dislocation interaction, $G$ is the bulk modulus of the Al alloy 3004 with value of $26 \mathrm{Gpa}$, while $b$ is the Burgers vector of Aluminum and equal $0.286 \mathrm{~nm}$.

The material hardness increases with the decreasing in the deformed region size: it is the ISE. The depth of indentation $(h)$ is related to GNDs, $\rho_{G N D}$, as follows [34]:

$$
\rho_{G N D}=\frac{3 \tan ^{2} \theta}{2 f^{3} b h}
$$

$\theta$ presents the angle between the surface of the material and the surface of the indenter, $b$ is the dislocations Burgers vector and $\mathrm{f}$ is the factor correction of the plastic zone size. In present work, $\theta=22^{\circ}, f=1.90$ [39] while $b=0.2860 \mathrm{~nm}$ 
[45]. A model was developed and a simplified deduced to give hardness and indentation depth relation [45]:

$$
\left(\frac{H}{H_{0}}\right)^{2}=1+h^{\star}\left(\frac{1}{h}\right)
$$

where $H$ is the hardness at a depth of indentation, $h, H_{0}$ is the hardness at the infinite depth and $h^{\star}$ is the characteristic length. $h^{\star}$ depends on the indenter shape and have value of $9 \times 10^{-4} \mathrm{~cm}$, as shown in Figure 7 .

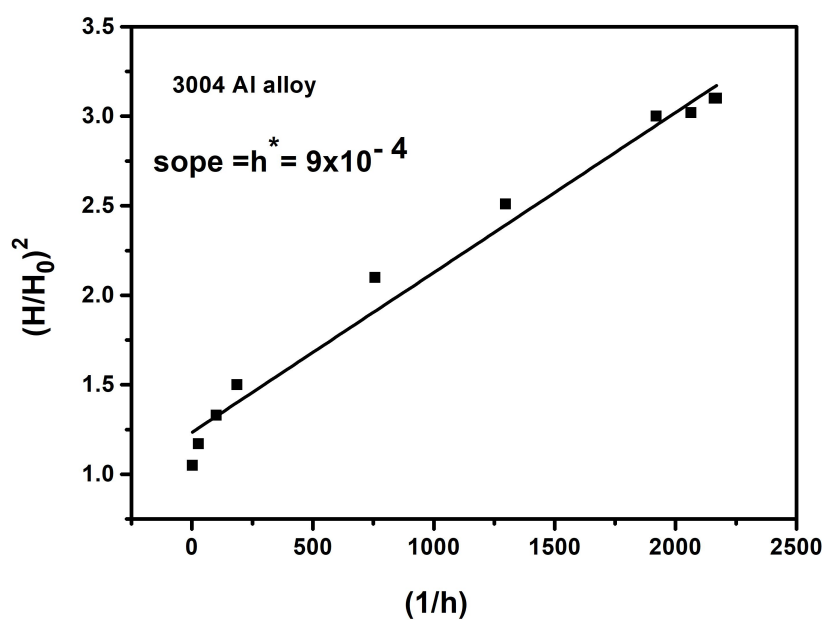

Figure 7: The ratio $\left(\frac{H}{H_{0}}\right)^{2}$ versus $\left(\frac{1}{h}\right)$ of the 3004 Al-alloy obtained by the Vickers hardness test

The dislocation density, $\rho$, and defect density, $\left(\rho^{-}\right)$, of the deformed $3004 \mathrm{Al}$-alloy were calculated by the Vickers hardness test and are shown in the figures. The relation between them is as follows [18]:

$$
\left(\rho^{-} \mathrm{cm}^{-3}\right)=\frac{\rho\left(\mathrm{cm}^{-2}\right)}{b}
$$

According to these equations, the total dislocation density, $\rho_{t}$, and the dislocation density due to GNDs, $\rho_{G N D}$, can be obtained. This is shown in Figure 8, where the dislocation density due to SSDs is: $\rho_{S S D}=4.9 \times 10^{9} \mathrm{~cm}^{-2}$.

The dislocation density in a metal increase with deformation or cold work, due to dislocation multiplication or the formation of new dislocations. Consequently, the average distance of separation between dislocations decreases, i.e., the dislocations are positioned closer together. On average, dislocation-dislocation strain interactions are repulsive. Obviously, the motion of dislocation is hindered by the presence of other dislocations. As the dislocation density increases, this resistance to dislocation motion by other dislocations becomes more pronounced. Clearly, the imposed stress necessary to deform a metal increase with increasing cold work.

The total defect density, $\rho_{t}^{-}$, and the defect density due to GNDs, $\rho_{G N D}^{-}$, can be obtained as shown in Figure 9, where the defect density due to SSDs is: $\rho_{S S D}{ }^{-}=1.65 \times 10^{17} \mathrm{~cm}^{-3}$.

Generally, the flow stress in terms of the shear stress resolved $(\tau)$ depend on the dislocation density root square as [44]:

$$
\tau=\alpha G b \sqrt{\rho}
$$

By using the above equation, the total flow stress, $\sigma_{t}$, and the flow stress due to GNDs, $\sigma_{G N D}$, can be obtained as shown in Figure 10, where the flow stress due to SSDs is: $\sigma_{S S D}=25.5 \mathrm{Mj} / \mathrm{m}^{3}$.

The micro-strain shown by the Vickers hardness test is shown in Figure 11. This exhibits an exponential increase with thickness reduction.

From the theory of dislocation, the stored dislocation energy (E) is based on the dislocation density, $\rho$, as follows [18]:

$$
E=\alpha G b^{2} \rho
$$
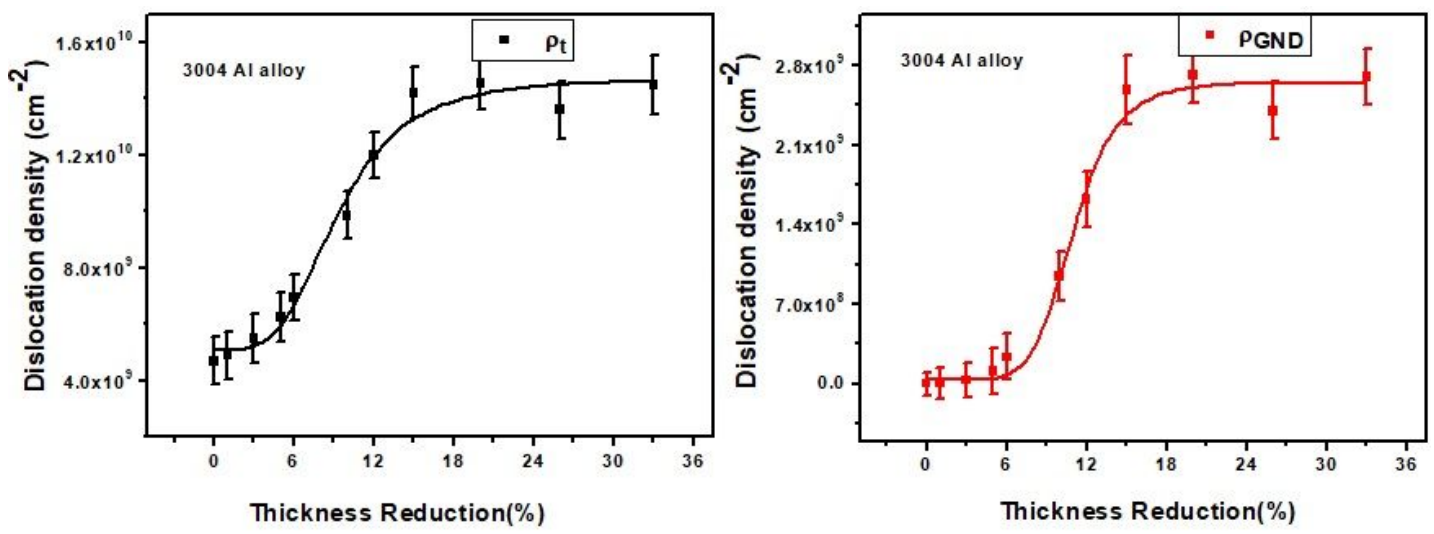

Figure 8: The dislocation density, $\rho_{t}$ and $\rho_{G N D}$, with the reduction of thickness for the $3004 \mathrm{Al}$-alloy by the Vickers hardness test 

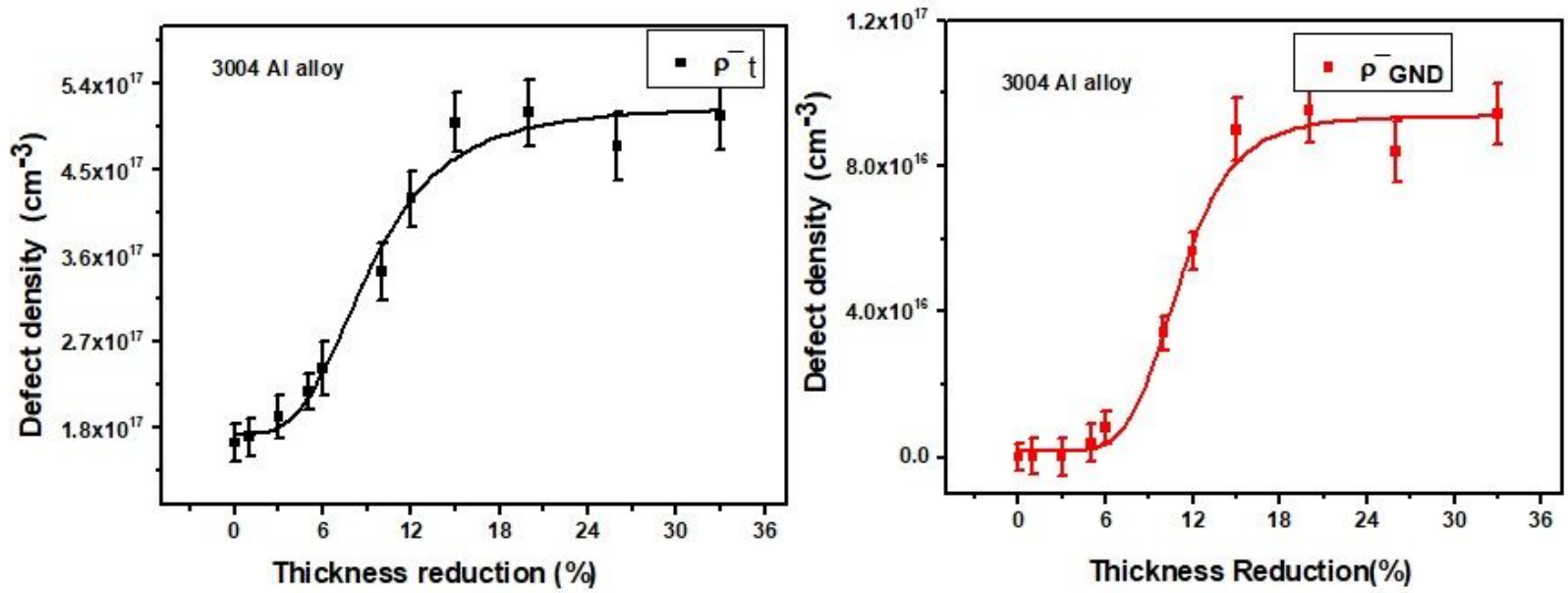

Figure 9: The defect density, $\rho_{t}{ }^{-}$and $\rho_{G N D}{ }^{-}$, as a function of the reduction of thickness for the Al-alloy 3004 with the Vickers hardness test
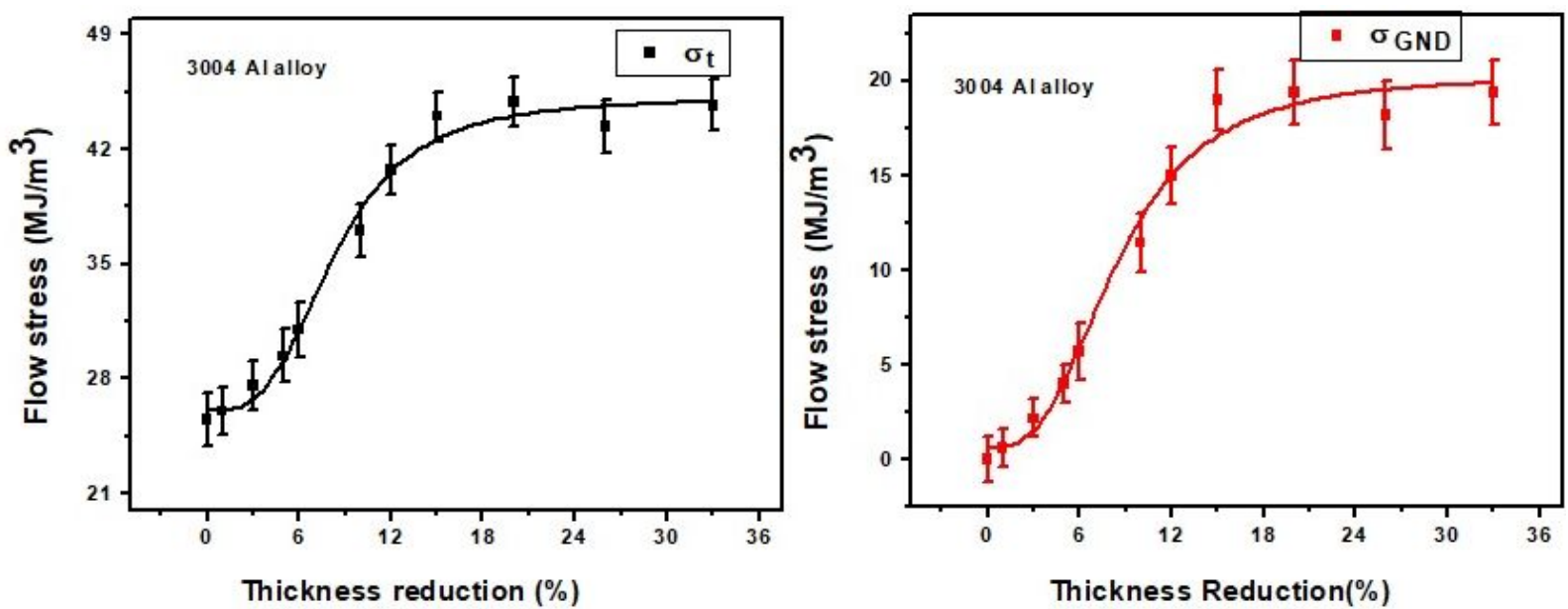

Figure 10: The flow stress, $\sigma_{t}$ and $\sigma_{G N D}$, with the reduction of thickness for the 3004 Al-alloy by the Vickers hardness test

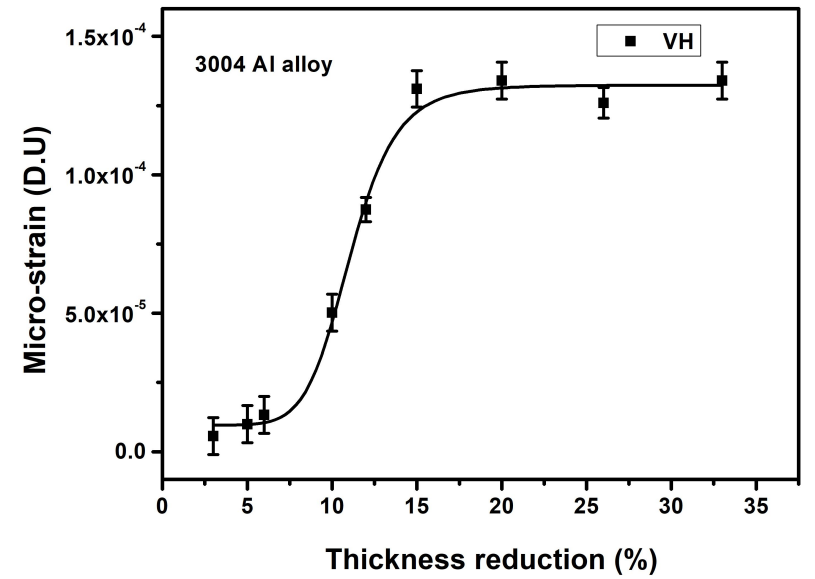

Figure 11: The micro-strain with the reduction of thickness for the $3004 \mathrm{Al}$-alloy obtained from the Vickers hardness test
According to the above relation, the total stored energy, $E_{t}$, and the energy stored due to GNDs, $E_{G N D}$, can be determined as shown in Figure 12, where the energy stored due to SSDs is: $E_{S S D}=50 \mathrm{kj} / \mathrm{m}^{3}$.

\subsection{Microstructural comparative between three techniques (PALT, PADBT and HV)}

As measured by PALT, PADBT and HV, the mean crystallite size exhibits an exponential decrease, as shown in Figure 13. A good correlation between the three techniques for the mean crystallite size is observed.

The defect density, dislocation density, stored dislocation energy flow stress and micro-strain were calculated with PALT, PADBT and HV. These exhibit an exponential increase, as shown in Figures 14-17, respectively. 

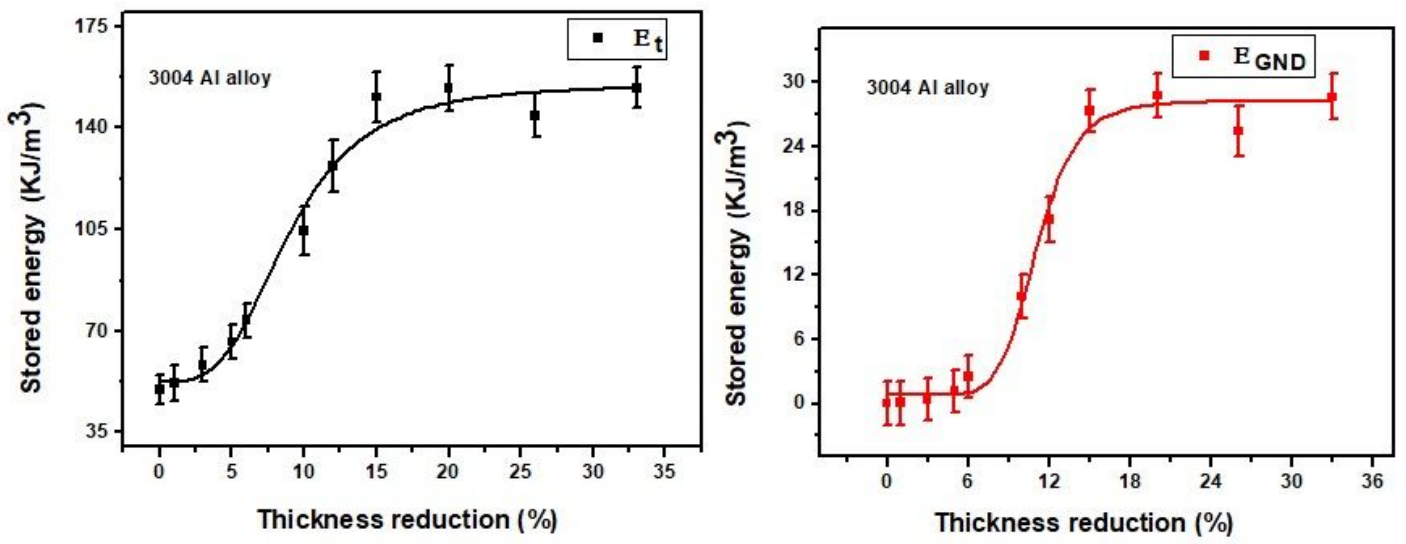

Figure 12: The stored energy, $E_{t}$ and $E_{G N D}$, of the deformed Al-alloy 3004 with the thickness obtained from the Vickers hardness test

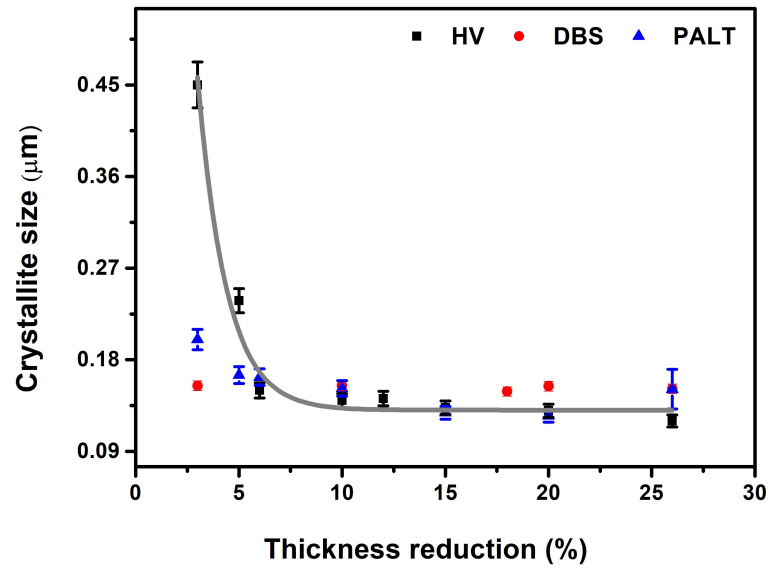

Figure 13: The crystallite size means with the reduction of thickness for the 3004 Al-alloy as measured with PALT, PADBT and HV

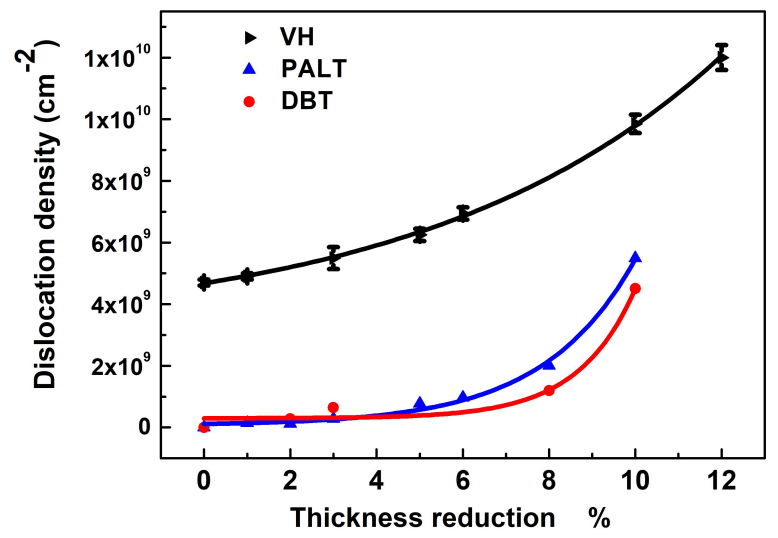

Figure 14: The dislocation density with the reduction of thickness for the $3004 \mathrm{Al}$-alloy as measured with PALT, PADBT and HV

A good correlation between PALT and PADBT is shown from 0 to $10 \%$ thickness reduction. $V_{H}$ has good correlations with PALT and PADBT from 0 to $6 \%$ thickness reduc-

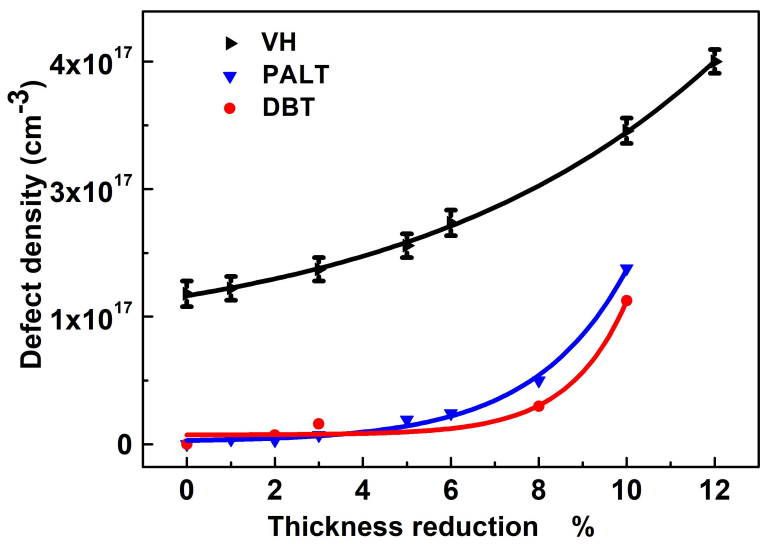

Figure 15: The defect density with the reduction of thickness for the 3004 Al-alloy as measured with PALT, PADBT and HV

tion: then a clear difference from 6 to 15\% thickness reduction is observed.

The values of crystallite size and other parameters as defect and dislocation density are different in values when using VHT compared to DBT and PALT. For example, the dislocation density values at different degrees of deformation by VHT are larger compared to DBT and PALT because they consider two additional dislocations SSDs (statically stored dislocations) and GNDs (Geometrically necessary dislocations) which arise from the indentation size effect (ISE). The same behavior is found for the other parameters such as micro strain, defect density, flow stress, and stored energy because they depend on the dislocation density.

Gomaa et al., 2005 investigate the Al-Mn alloy (3004) isochronal annealing and conclude that there is a positive correlation between the average positron's lifetime $\tau_{a v}$ and microhardness $H_{v}$. In addition, at the high cold work increasing recrystallization process and the low cold work increasing the recovery process [46]. 

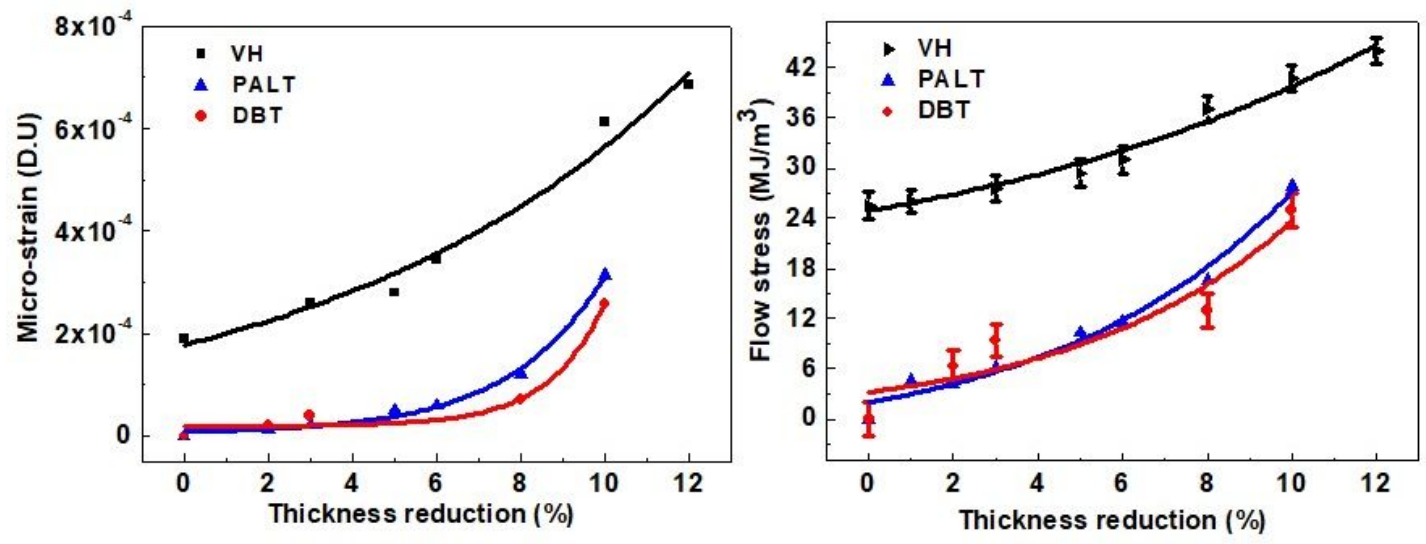

Figure 16: Micro-strain and flow stress with the thickness reduction of the Al-alloy 3004 as measured with PALT, PADBT and HV

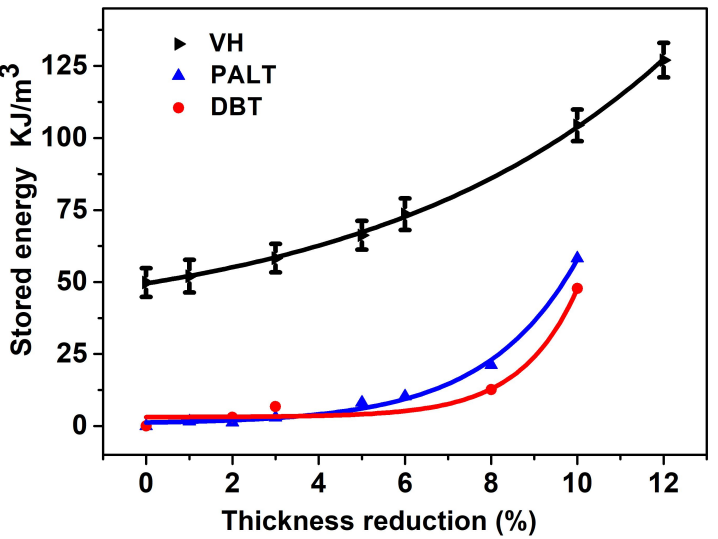

Figure 17: The stored energy with the reduction of thickness for the 3004 Al-alloy as measured with PALT, PADBT and HV

\section{Conclusion}

Based on the obtained results from the PALT, PADBT and HV measurements on the 3004 wrought aluminum alloy at different degrees of thickness reduction, the following conclusions can be presented.

The mean parameters (micro-strain and crystallite size) were obtained for deformed and non-deformed samples with PALT. The mean crystallite size seems to exponentially decrease with increasing of thickness reduction, while the micro-strain undergoes exponential growth. Other parameters such defect density, dislocation density, micro-strain and stored dislocation energy were obtained from the Sand W-parameters with PADBT. These parameters showed exponential growth. The obtained parameters from PALT and PADBT are compared with those obtained from HV. A good correlation between the three techniques (PALT, PADBT and HV) for mean crystallite size was found. PALT, PADBT and HV produce different values for defect density, dislocation density, micro-strain, and stored dislocation energy after 6 to $15 \%$ thickness reduction.

The dislocation density and defect density values at different deformations, determined by VHT, are larger as compared to the values obtained by the other used experimental techniques because they result due to two types of dislocations, the Statically Stored Dislocations (SSDs), and the Geometrically Necessary Dislocations (GNDs).

Funding information: The authors state no funding involved

Author contributions: All authors have accepted responsibility for the entire content of this manuscript and approved its submission.

Conflict of interest: The authors state no conflict of interest.

\section{References}

[1] Sajuri Z, Selamat NF, Baghdadi AH, Rajabi A, Omar MZ, Kokabi AH, et al. Cold-rolling strain hardening effect on the microstructure, serration-flow behaviour and dislocation density of friction stir welded AA5083. Metals (Basel). 2020;10(1):10.

[2] Li G, Lu H, Hu X, Lin F, Li X, Zhu Q. Current progress in rheoforming of wrought aluminum alloys: A review. Metals (Basel). 2020;10(2):10.

[3] Jaradeh MM. The Effect of Processing Parameters and Alloy Composition on the Microstructure Formation and Quality of DC Cast Aluminium Alloys [dissertation]. Sundsvall: Mid Sweden University; 2006.

[4] 'Sayed Ahmed' EMH. Characterization of Control Mesoporous Glasses (CPGs) Using Positron Annihilation Lifetime Spectroscopy (PALS) [dissertation]. Halle-Wittenberg: Martin Luther University; 2001. 
[5] Fong C, Dong AW, Hill AJ, Boyd BJ, Drummond CJ. Positron annihilation lifetime spectroscopy (PALS): a probe for molecular organisation in self-assembled biomimetic systems. Phys Chem Chem Phys. 2015 Jul;17(27):17527-40.

[6] Bokov AV, Byakov VM, Kulikov LA, Perfiliev YD, Stepanov SV. To the application of the emission Mössbauer and positron annihilation spectroscopies for detection of carcinogens. Hyperfine Interact. 2017;238(1):238.

[7] Banhart J, Liu M, Yong Y, Liang Z, Chang CS, Elsayed M, et al. Study of ageing in Al-Mg-Si alloys by positron annihilation spectroscopy. Physica B. 2012;407(14):2689-96.

[8] Axpe E, Lopez-Euba T, Castellanos-Rubio A, Merida D, Garcia JA, Plaza-Izurieta $\mathrm{L}$, et al. Detection of atomic scale changes in the free volume void size of three-dimensional colorectal cancer cell culture using positron annihilation lifetime spectroscopy. PLoS One. 2014 Jan;9(1):e83838.

[9] Moskal P, Kisielewska D, Curceanu C, Czerwiński E, Dulski K, Gajos A, et al. Feasibility study of the positronium imaging with the J-PET tomograph. Phys Med Biol. 2019;64:055017.

[10] Eren B, Eren E, Guney M, Jean YC, Van Horn JD. Positron annihilation lifetime spectroscopy study of polyvinylpyrrolidone-added polyvinylidene fluoride membranes: investigation of free volume and permeation relationships. J Polym Sci. 2020;58(4):589-98.

[11] Tuomisto F, Makkonen I. Defect identification in semiconductors with positron annihilation: experiment and theory. Rev Mod Phys. 2013;85(4):1583-631.

[12] Do Nascimento E, Vanin VR, Maidana NL, Helene O. Coincidence doppler broadening of positron annihilation radiation in Fe. J Phys Conf Ser. 2013;443:443.

[13] do Nascimento E, Helene O, Vanin VR, da Cruz MT, Moralles M. Statistical analysis of the Doppler broadening coincidence spectrum of electron-positron annihilation radiation in silicon. Nucl Instrum Methods Phys Res A. 2009;609(2-3):244-9.

[14] Do Nascimento E, Helene O, Takiya C, Vanin VR. Doppler broadening of positron annihilation radiation: fitting the coincidence spectrum. Nucl Instrum Methods Phys Res A. 2005;538(1-3):72330.

[15] Wagner A, Butterling M, Liedke MO, Potzger K, KrauseRehberg R. Positron annihilation lifetime and Doppler broadening spectroscopy at the ELBE facility. AIP Conf Proc. 2018;1970(1):040003.

[16] Tsapko EA, Galstian IY. Positron spectroscopy study of structural defects and electronic properties of carbon nanotubes. Prog Phys Met. 2020;21:153-79.

[17] Badawi EA, Abdel-Rahman MA, Salah M, Abdel-Rahman M. Study the effect of plastic deformation in 8006 Al-alloys by Positron lifetime spectroscopy, Vickers hardness and X-ray diffraction. Defect Diffus Forum. 2016;373:142-5.

[18] Badawi EA, Abdel-Rahman MA, El-Nahhas AA. Correlation coefficient between vickers hardness and nuclear technique. Defect Diffus Forum. 2013;344:129-36.

[19] Imbrogno S, Umbrello D, Schulze V, Zanger F, Segebade E. Metallurgical and material properties correlations between machined and severely plastic deformed aluminium alloy. Int J Mater Form. 2020;13(5):699-708.

[20] Kapoor G, Kvackaj T, Heczel A, Bidulská J, Kočiško R, Fogarassy Z, et al. The influence of severe plastic deformation and subsequent annealing on the microstructure and hardness of a $\mathrm{Cu}-\mathrm{Cr}-\mathrm{Zr}$ alloy. Materials (Basel). 2020 May;13(10):13.
[21] Ojdanic A, Horky J, Mingler B, Fanetti M, Gardonio S, Valant M, et al. The Effects of Severe Plastic Deformation and/or Thermal Treatment on the Mechanical Properties of Biodegradable MgAlloys. Metals. 2020;10(8):1-32.

[22] Panigrahi A, Sulkowski B, Waitz T, Ozaltin K, Chrominski W, Pukenas $A$, et al. Mechanical properties, structural and texture evolution of biocompatible $\mathrm{Ti}-45 \mathrm{Nb}$ alloy processed by severe plastic deformation. J Mech Behav Biomed Mater. 2016 Sep;62:93-105.

[23] Tiryakioğlu M, Robinson JS. On the representative strain in Vickers hardness testing of 7010 aluminum alloy. Mater Sci Eng A. 2015;641:231-6.

[24] Merayo D, Rodríguez-Prieto A, Camacho AM. Prediction of mechanical properties by artificial neural networks to characterize the plastic behavior of aluminum alloys. Materials (Basel). 2020 Nov;13(22):1-22.

[25] Tiryakioğlu M, Robinson JS, Salazar-Guapuriche MA, Zhao YY, Eason PD. Hardness-strength relationships in the aluminum alloy 7010. Mater Sci Eng A. 2015;631:196-200.

[26] Sekhar AP, Nandy S, Ray KK, Das D. Prediction of Aging Kinetics and Yield Strength of 6063 Alloy. J Mater Eng Perform. 2019;28(5):2764-78.

[27] Tiryakioğlu M, Robinson JS. On the representative strain in Vickers hardness testing of 7010 aluminum alloy. Mater Sci Eng A. 2015;641:231-6.

[28] Badawi E, Abdel-Rahman M, Attallah A, Abdel-Nasser M. Defect Investigation of Some Commercial Aluminum Alloys by Doppler Broadening Technique. Egypt J Phys. 2017;45:23-8.

[29] Abd-Elhakim MH, Mostafa M, Darwash M, Abdel-Rahman M, Abdel-Rahman MA, Badawi EA. Probing properties of 6061 aluminum alloy used as cladding for nuclear reactor fuel with X-ray diffraction. AIP Conf Proc. 2020;2313(1):060021.

[30] Badawi EA, Abdel-Naser M, Ahmed AA. Influence of plastic deformation on the properties of 6066 heat treatable aluminum alloys. Walailak J Sci Technol. 2013;10:289-95.

[31] Hautakangas S, Schut H, Van Der Zwaag S, Del Castillo PE, Van Dijk NH. Positron annihilation spectroscopy as a tool to develop self healing in aluminium alloys. Phys Status Solidi (C) Curr Top Solid State Phys. 2007;4:3469-72.

[32] Coleman P. Positron beams and their applications. London: World Scientific; 2000. p. 41-87 https://doi.org/10.1142/3719.

[33] Chandler H. Hardness Testing. $2^{\text {nd }}$ edition. United States; 1999.

[34] Li J, Zhang M, Li B, Monteiro SN, Ikhamyies S, Kalay YE, et al. Characterization of Minerals, Metals, and Materials. Cham: Springer; 2017.

[35] Totten GE, Newkirk JW, Funatani K, Solari M, Xie L, Xun G, et al. Handbook of Metallurgical Process Design. Boca Raton (FL): CRC Press; 2004. https://doi.org/10.1201/9780203970928.

[36] Yang B, Vehoff H. Dependence of nanohardness upon indentation size and grain size - A local examination of the interaction between dislocations and grain boundaries. Acta Mater. 2007;55(3):849-56.

[37] Wang Y, Tomota Y, Ohmura T, Gong W, Harjo S, Tanaka M. Continuous and discontinuous yielding behaviors in ferrite-cementite steels. Acta Mater. 2020;196:565-75.

[38] Durst K, Franke O, Böhner A, Göken M. Indentation size effect in Ni-Fe solid solutions. Acta Mater. 2007;55(20):6825-33.

[39] Durst K, Backes B, Franke O, Göken M. Indentation size effect in metallic materials: modeling strength from pop-in to macroscopic hardness using geometrically necessary dislocations. Acta Mater. 2006;54(9):2547-55. 
[40] Nix WD, Gao H. Indentation size effects in crystalline materials: A law for strain gradient plasticity. J Mech Phys Solids. 1998;46(3):411-25.

[41] Abdel-Rahman M. Detecting thermal defects in age hardening Al-6063 alloys by positron annihilation Doppler broadening technique. Phys Status Solidi (C) Curr Top Solid State Phys. 2009;6:2359-63.

[42] Waseem OA, Woller KB. Evolution in microstructure and hardness of Titanium-Zirconium-Molybdenum (TZM) alloy after depth marker implantation for erosion diagnostic in fusion devices. Mater Chem Phys. 2021;258:123883.

[43] Jiang C, Gao Q, Zhang H, Liu Z, Li H. Microstructure and mechanical properties of 4 al alumina-forming austenitic steel after cold-rolling deformation and annealing. Materials (Basel). 2020 Jun;13(12):1-18.
[44] Dini G, Ueji R, Najafizadeh A, Monir-vaghefi SM. Flow stress analysis of TWIP steel via the XRD measurement of dislocation density. Mater Sci Eng A. 2010;527(10-11):2759-63.

[45] Vasilev D. Alternative Approaches to Measure a Dislocation Density. Physical Metallurgy. 2012.

[46] Gomaa E, Mohsen M, Taha AS, Mostafa MM. A study of annealing stages in Al-Mn (3004) alloy after cold rolling using positron annihilation lifetime technique and Vickers microhardness measurements. Mater Sci Eng A. 2003;362(1-2):274-9. 